\title{
POST-RADIATION SARCOMA IN ANKYLOSING SPONDYLITIS
}

A Report of Five Cases

\author{
M. A. Edgar, London, and M. P. Robinson, Oswestry, England \\ From the Robert Jones and Agnes Hunt Orthopaedic Hospital, Oswestry
}

The carcinogenic properties of roentgen rays were recognised a few years after their discovery, and in 1922 Beck reported the first instances of sarcoma developing in patients previously treated with radiotherapy. More recent reviews of sarcoma following bone irradiation include those of Sabanas, Dahlin, Childs and Ivins (1956), Phillips and Sheline (1963), Steiner (1965), Dahlin and Coventry (1967) and Hatfield and Schulz (1970). These reports reveal the extremely malignant nature of post-radiation sarcomas.

The use of radiotherapy as an effective pain-reliever in ankylosing spondylitis has been appreciated for many years (Hair 1954). However, Court-Brown and Doll (1957) drew attention to the alarming increase in leukaemia among those who had been irradiated for this condition. "For all patients the annual incidence rises to 72 cases per 10,000 following a mean dose to the spinal marrow in excess of 2,250 r." They calculated the annual incidence among those not given therapeutic irradiation to be 0.5 case per 10,000 .

Their later study (Court-Brown and Doll 1965) revealed an increased incidence of various types of malignant tumours arising in heavily irradiated tissues. Although they were able to trace only two confirmed cases of bone sarcoma, they concluded that "mortality attributable to radiation may increase twofold or more with the passage of time".

Three more sarcomas have now occurred (Cases 1, 2 and 5) and these are reported below with the two previously known cases from Sir Richard Doll's series (Cases 3 and 4). The patients in Cases 1 and 2 were recently under our care at Oswestry. The woman in Case 5 was under the care of Mr G. V. Osborne of Liverpool.

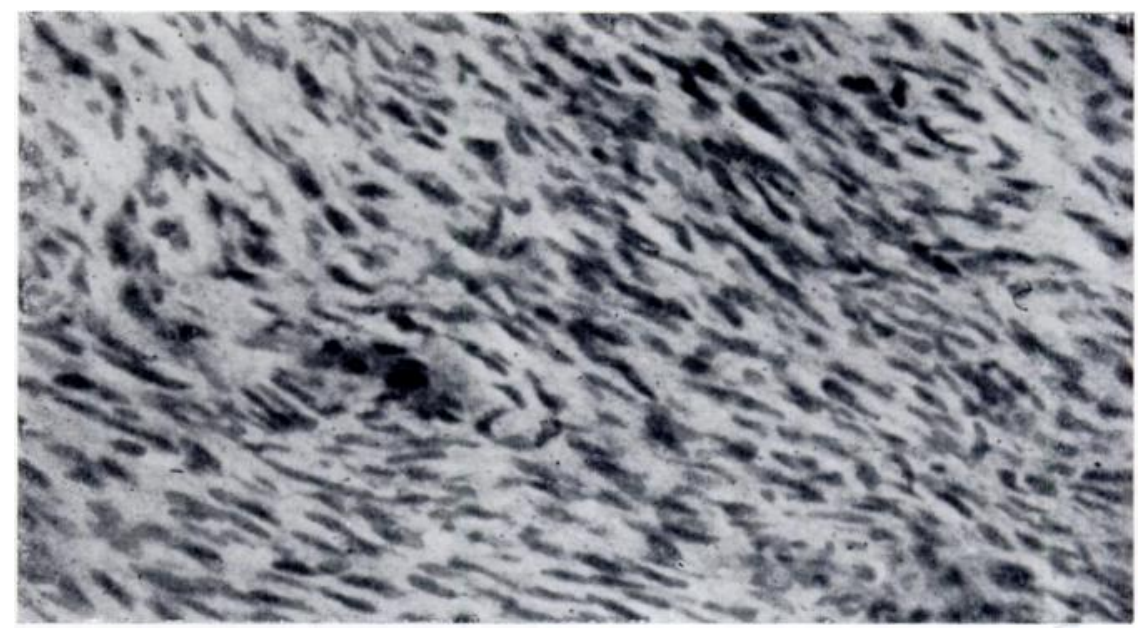

Fig. 1

Case 1-Photomicrograph of a section showing undifferentiated fibrosarcoma. (Haematoxylin and eosin, $\times 150$.)

\section{CASE REPORTS}

Case 1-A man aged twenty-six developed ankylosing spondylitis in 1948. His symptoms were initially controlled with salicylates, but after a relapse he was referred for radiotherapy 
in December 1953. The sacro-iliac region, thoraco-lumbar spine and thoraco-cervical spine were each given a skin dose of $1,800 \mathrm{r}$ in six treatments over a period of three weeks, using 230 kilovolts, $15 \mathrm{~mA}$ through a 2.6-millimetre $\mathrm{Cu}$ half value layer filter. After this he improved and remained well, apart from a short recurrence of pain in 1958.

In May 1968 he developed right sciatica with paresis of the right hip and knee muscles which rapidly progressed. The erythrocyte sedimentation rate was 2 millimetres in the first hour (Wintrobe) and the serum alkaline phosphatase 15 K.A. units. Plain radiographs of the lumbar spine and pelvis showed only the typical changes of ankylosing spondylitis. Lumbar myelography revealed a spinal block at the level of the second lumbar vertebra, the appearances being consistent with an intrathecal neoplasm. Exploration confirmed the presence of a tumour which involved the dura anteriorly and infiltrated intrathecally around the cauda equina. The tumour was incompletely excised. Histology showed an undifferentiated spindle-cell fibrosarcoma (Fig. 1); the tissue of origin could not be determined. After operation there was some neurological improvement for three months. Eventually the tumour caused a complete cauda equina lesion and in March 1969 he died from pulmonary metastases.

At necropsy we found a massive tumour of the vertebral bodies from T.12 to L.4, with paravertebral extension. Histology confirmed the previous findings.

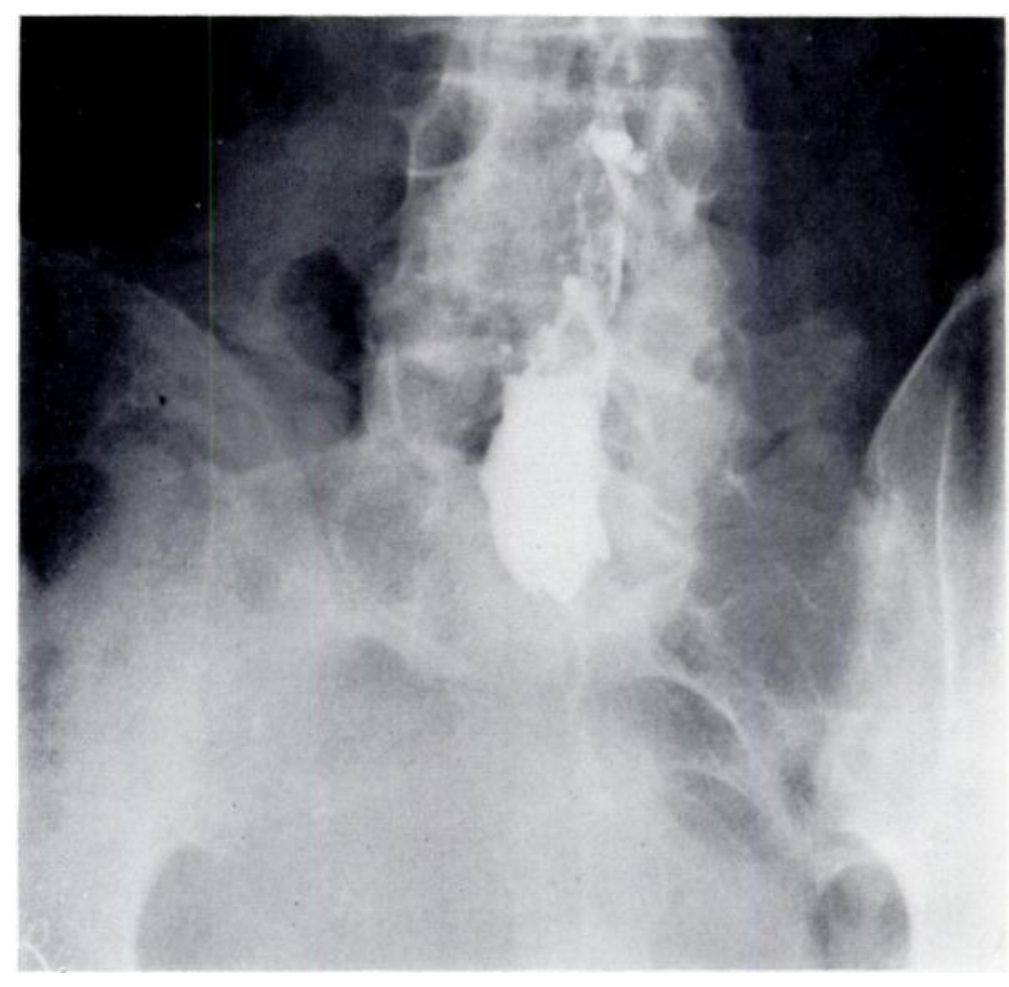

Fig. 2

Case 2-Radiograph of the sacrum after myelography, showing erosion of the right side with loss of definition of the sacral foramina.

Case 2-A man aged nineteen was treated with radiotherapy in July 1952 after ankylosing spondylitis had been diagnosed. Irradiation was given to the entire spine in three fields and the sacro-iliac joints, each field receiving 1,360 r. The right hip in two fields and the knee each received 1,600 r. Treatment was given in fourteen sessions over three weeks, using 230 kilovolts, $15 \mathrm{~mA}$ with a 1.4-millimetre $\mathrm{Cu}$ half value layer filter, and was terminated because of a fall in the white-cell count to 1,000 per cubic millimetre. 
He remained symptom-free until November 1969 when he was referred by his general practitioner with a recurrence of lumbo-sacral pain and an erythrocyte sedimentation rate of 70 millimetres in the first hour. The pain improved with analgesics. A further erythrocyte sedimentation rate was 6 millimetres in the first hour (Wintrobe). Three months later he was again referred with a cauda equina lesion. He was admitted to Oswestry, at which time the erythrocyte sedimentation rate was 7 millimetres in the first hour (Wintrobe) and the serum alkaline phosphatase normal. Plain radiographs showed extensive osteolysis in the upper sacrum on the right side (Fig. 2). Lumbar myelography was normal, but the protein content of the cerebrospinal fluid was raised to 160 milligrams per cent. At operation, removal of the fifth lumbar and first sacral laminae revealed a large anterior sacral tumour. Histological examination of the biopsy specimen showed it to be an undifferentiated fibrosarcoma having a whorled pattern. The tissue of origin was not determined.

After a transient improvement following decompression the patient's general condition gradually deteriorated and he died in May 1970. Permission for post-mortem examination was refused.

Case 3-A man aged forty-one presented in September 1945 with back pain, painful heels and iritis: a diagnosis of ankylosing spondylitis was made. His symptoms were relieved by radiotherapy. The first course of treatment consisted of a total skin dose of $2,000 \mathrm{r}$ given to the cervical and thoracic spine in five sessions over a period of four weeks, using 200 kilovolts, $8 \mathrm{~mA}$ with a 1.6-millimetre, 1-millimetre aluminium filter. In 1952 another course of treatment was given to the whole spine and sacro-iliac region, using 250 kilovolts, $18 \mathrm{~mA}$, a total skin dose of $2,400 \mathrm{r}$ being delivered in three treatments over a period of five days. The sacro-iliac and lumbar spine fields each received $600 \mathrm{r}$.

The disease remained quiescent until August 1960 when a painful lump appeared above the left ischial tuberosity. Radiographs showed erosion of the left ischium and multiple pulmonary metastases. Biopsy of the buttock mass showed it to be a fibrosarcoma or leiomyosarcoma.

The patient died three weeks later in January 1961 from sarcomatosis. Necropsy failed to establish the tissue of origin.

Case 4-A man aged eighteen presented in 1952 with back pain and stiffness of the hips. Ankylosing spondylitis was diagnosed and he was treated with radiotherapy. The whole spine (in three fields), the sacro-iliac region and the right hip received a total skin dose of 11,600 $\mathrm{r}$ using 190 kilovolts, $6 \mathrm{~mA}$ with a 1·09-millimetre $\mathrm{Cu}$ half value layer filter. The lumbar spine and the sacro-iliac area each received 2,000 $\mathrm{r}$ and the right hip received 3,600 $\mathrm{r}$ through anterior and posterior fields. This course was administered in daily treatments over a period of four weeks. Five months later $3,600 \mathrm{r}$ were given to the left hip in eight daily treatments. His symptoms were completely relieved.

During 1961 he developed right sciatica which later became associated with extensive motor and sensory changes in the right leg. A hard fixed mass was palpated deep in the right iliac fossa. Radiographs showed only the typical changes of ankylosing spondylitis. At operation a large tumour infiltrating the right iliacus and lumbo-sacral plexus was removed through a retroperitoneal approach. The histological diagnosis was a spindle-cell fibrosarcoma. The tissue of origin was not suggested.

$\mathrm{He}$ was given palliative irradiation and died at home six months later.

Case 5-A woman aged forty-seven was seen in 1949 and a diagnosis of ankylosing spondylitis was made. A course of radiotherapy was given to the whole spine (three fields) and the sacro-iliac region. A total skin dose of 9,410 $\mathrm{r}$ was administered in ten treatments over two weeks using 2,000 kilovolts, $15 \mathrm{~mA}$ with a 2-millimetre copper half value layer filter. The sacro-iliac field received a skin dose of $2,260 \mathrm{r}$. This treatment was followed by a remission of symptoms until 1951 when a further course of irradiation totalling 5,500 $\mathrm{r}$ was given to the 
spine, sacro-iliac region and left hip under similar conditions, the sacro-iliac region receiving $1,000 \mathrm{r}$ in ten treatments over two weeks. Satisfactory relief of her symptoms occurred. In 1952 a further bout of pain was relieved with cortisone.
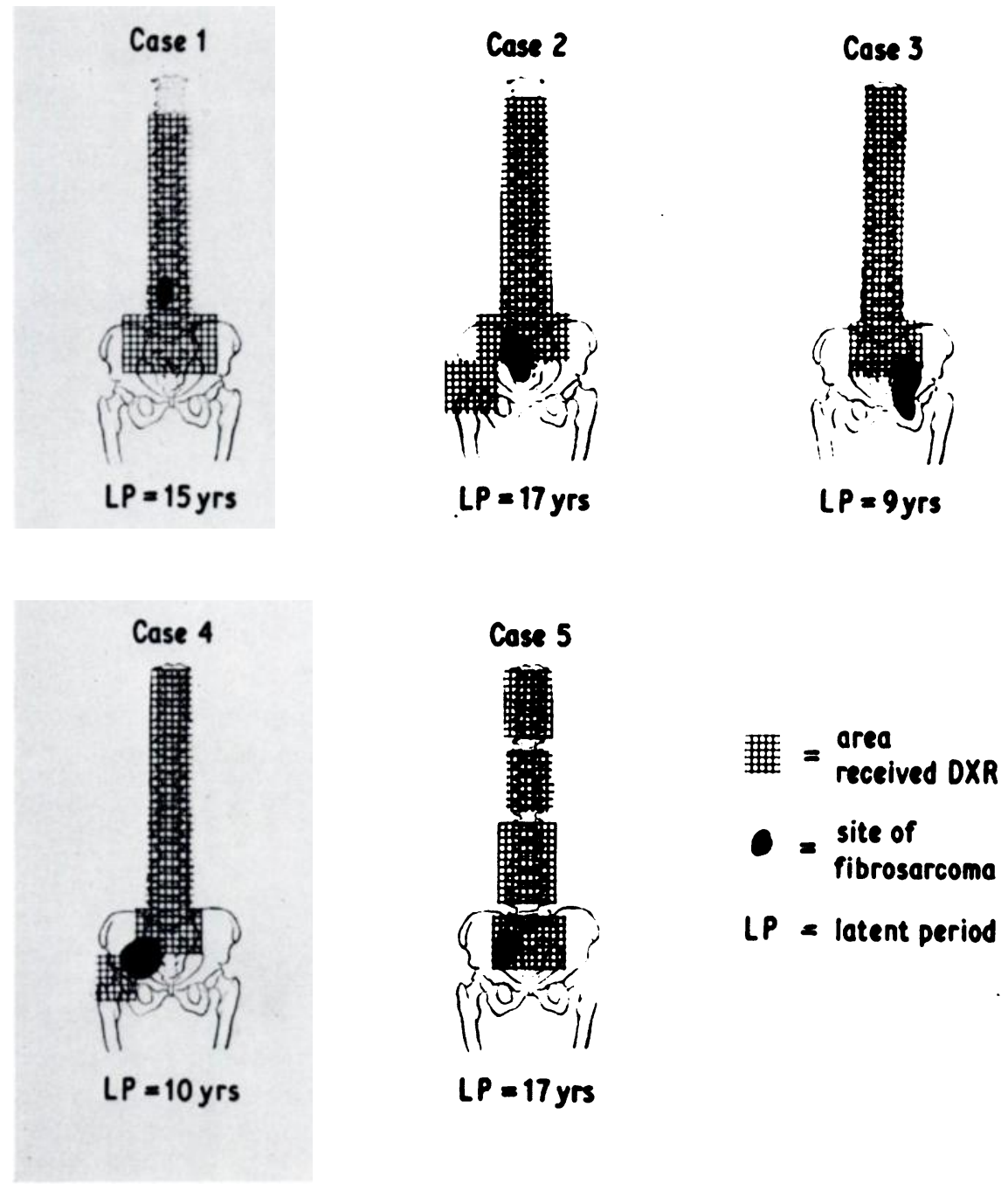

Diagrammatic representation of the areas irradiated and site of fibrosarcoma in each case.

In 1966 a painful swelling over the right sacro-iliac joint appeared, and radiographs showed a destructive lesion of the sacrum and a metastasis in the right lung field. Biopsy of the sacro-iliac swelling was performed and the histology revealed a poorly differentiated fibrosarcoma. Palliative irradiation was given and she died three months later. No conclusion was reached about the tissue of origin of the tumour. A review of this patient's radiographs cast some doubt on the original diagnosis of ankylosing spondylitis in 1949.

\section{DISCUSSION}

Histological examination of the tumours in each of these cases revealed an undifferentiated fibrosarcoma (Fig. 1). In their review of forty-three post-radiation sarcomas Dahlin and Coventry (1967) found eighteen fibrosarcomas and these formed the largest histological group. 
The site of origin of the fibrosarcoma was not confirmed in any of the patients in this series. In Cases 2, 3 and 5 bony erosion was present in the early radiographs and therefore a bony origin was suggested, although the possibility that the tumour was a leiomyosarcoma was considered in Case 3. A soft-tissue origin to the sarcoma seems more likely in Cases 1 and 4, although post-mortem examination in the former revealed extensive bony destruction. These tumours, with their extremely aggressive behaviour, differ from the fibrosarcomas of bone reviewed by Eyre-Brook and Price (1969). Perhaps this difference is related to their irradiation etiology and is not necessarily inconsistent with the tissue of origin being bone.

TABLE I

InCidence of Objective Neurological Signs in the Lower Limbs in 290 Cases of ANkylosing Spondylitis, Treated With and WITHOUT IRRADIATION, SEEN AT OSWESTRY FROM 1940 TO 1970

\begin{tabular}{|lccccc|}
\hline Cause of neurological lesion & & \multicolumn{2}{c|}{$\begin{array}{c}\text { Number of cases } \\
\text { Irradiated } \\
\text { (139) }\end{array}$} & $\begin{array}{c}\text { Not irradiated } \\
\mathbf{( 1 5 1 )}\end{array}$ \\
\hline Spinal trauma & $\cdot$ & $\cdot$ & $\cdot$ & 3 & 3 \\
Spinal osteotomy & $\cdot$ & $\cdot$ & $\cdot$ & 2 & 0 \\
Spinal tumour & $\cdot$ & $\cdot$ & $\cdot$ & 2 & 0 \\
Atlanto-axial subluxation &. & 0 & 1 \\
\hline
\end{tabular}

Cahan, Woodard, Higinbotham, Stewart and Coley (1948) laid down four criteria to be fulfilled before a sarcoma could be considered as a post-radiation tumour: 1) the initial bone lesion which was treated by radiotherapy should be benign;2) an asymptomatic latent period should follow irradiation; 3) the sarcoma should arise within the irradiated area; and 4) the tumour should be histologically confirmed.

The case histories reported above fulfil these criteria (Fig. 3). It might be considered that the latent period of seventeen years in Cases 2 and 5 is unusually long. However, latent periods as high as thirty years have been reported (Sabanas et al. 1956).

Three patients (Cases 1, 2 and 4) presented with sciatic pain accompanied by marked neurological signs. The other two patients presented with buttock pain. The clinical picture in all patients was thought at first to be related to an exacerbation in the ankylosing spondylitis. However, in retrospect, the assumption that neurological abnormalities can arise spontaneously in ankylosing spondylitis needs to be questioned.

In order to investigate the incidence of abnormal neurological physical signs in ankylosing spondylitis, the case records of 290 such patients seen at Oswestry during the last thirty years were reviewed. Only eleven cases were found in which there were objective neurological signs in the lower limbs (Table I). All of these were associated with serious complications of the disease or other pathology.

It is therefore reasonable to conclude that acute neurological abnormalities occurring "spontaneously" after a latent period following radiotherapy for ankylosing spondylitis should prompt investigation for an underlying spinal or paraspinal sarcoma.

The object of this paper is not to detract from the benefit which radiotherapy has provided for the majority of ankylosing spondylitis patients (Hair 1954). It is nevertheless salutary to balance this benefit against the already known, yet uncommon, complications which have been added to in this report.

VOL. 55 B, NO. 1, FEBRUARY 1973 


\section{SUMMARY}

1. Five cases of fibrosarcoma arising after radiotherapy in cases of ankylosing spondylitis are reported.

2. The relationship of the tumours to irradiation is discussed.

3. Objective neurological signs arising in the lower limbs of patients with ankylosing spondylitis after a latent period following radiotherapy may indicate an underlying sarcoma.

We wish to thank Sir Richard Doll for his help with this study and for his permission to report Cases 3 and 4 from his series. We are grateful to Dr George Meachim and Mr G. V. Osborne of Liverpool for allowing us to report Case 5. Dr P. Haslem of Clatterbridge kindly gave assistance with the technical details of radiotherapy.

\section{REFERENCES}

BECK, A. (1922): Zur Frage des Röntgensarkoms, zugleich ein Beitrag zur Pathogenese des Sarkoms. Münchener medizinische Wochenschrift, 69, 623.

Cahan, W. G., Woodard, H. Q., Higinbotham, N. L., Stewart, F. W., and Coley, B. L. (1948): Sarcoma Arising in Irradiated Bone. Cancer, 1, 3.

Court-Brown, W. M., and Doll, R. (1957): Leukaemia and Aplastic Anaemia in Patients Irradiated for Ankylosing Spondylitis. Medical Research Council, Special Report Series No. 295. London: H.M. Stationery Office.

Court-Brown, W. M., and Doll, R. (1965): Mortality from Cancer and other Causes after Radiotherapy for Ankylosing Spondylitis. British Medical Journal, 2, 1327.

Dahlin, D. C., and Coventry, M. B. (1967): Osteogenic Sarcoma. Journal of Bone and Joint Surgery, 49-A, 101.

Eyre-Brook, A. L., and Price, C. H. G. (1969): Fibrosarcoma of Bone. Journal of Bone and Joint Surgery, 51-B, 20.

HaIR, J. A. G. (1954): Treatment of Ankylosing Spondylitis by Radiotherapy. Journal of Bone and Joint Surgery, 36-B, 671.

Hatfield, P. M., and Schulz, M. D. (1970): Post-irradiation Sarcoma. Radiology, 96, 593.

Phillips, T. L., and Sheline, G. E. (1963): Bone Sarcoma following Radiation Therapy. Radiology, $81,992$.

Sabanas, A. O., Dahlin, D. C., Childs, D. S., Jun., and Ivins, J. C. (1956): Post-radiation Sarcoma of Bone. Cancer, 9, 528.

SteIner, G. C. (1965): Post-irradiation Sarcoma of Bone. Cancer, 18, 603. 